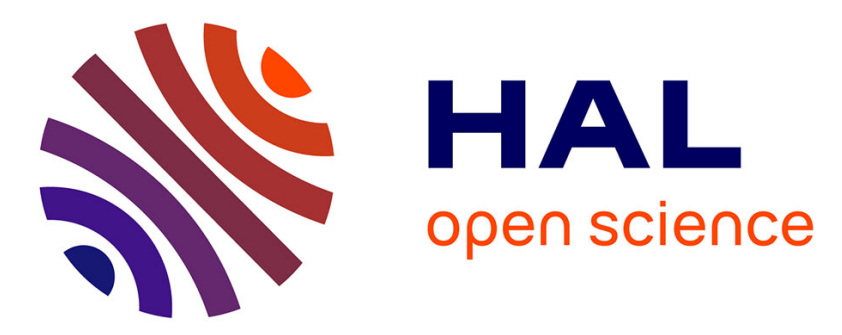

\title{
La prospective territoriale, révélateur et outil d'une action publique territorialisée
}

Marie-Christine Fourny, Damien Denizot

\section{To cite this version:}

Marie-Christine Fourny, Damien Denizot. La prospective territoriale, révélateur et outil d'une action publique territorialisée. Rodolphe Dodier; Alice Rouyer; Raymonde Séchet. Territoires en action et dans l'action, Presses Universitaires de Rennes, pp.29-44, 2007. halshs-00136964

\section{HAL Id: halshs-00136964 https://shs.hal.science/halshs-00136964}

Submitted on 18 Mar 2008

HAL is a multi-disciplinary open access archive for the deposit and dissemination of scientific research documents, whether they are published or not. The documents may come from teaching and research institutions in France or abroad, or from public or private research centers.
L'archive ouverte pluridisciplinaire HAL, est destinée au dépôt et à la diffusion de documents scientifiques de niveau recherche, publiés ou non, émanant des établissements d'enseignement et de recherche français ou étrangers, des laboratoires publics ou privés. 


\title{
La prospective territoriale, révélateur et outil d'une action publique territorialisée
}

\author{
In Territoires en action et dans l'action. Sous la direction de Rodolphe Dodier, Alice \\ Rouyer, Raymonde Séchet. Presses universitaires de Rennes. 2007, p. 29-44
}

\author{
Marie-Christine FOURNY et Damien DENIZOT \\ Institut de Géographie Alpine, Université Joseph Fourier (Grenoble I) \\ UMR PACTE, Laboratoire Territoires \\ 14 bis, av. Marie Reynoard \\ 38100 Grenoble \\ Fourny-Kober.Marie-Christine@ujf-grenoble.fr \\ denizot.damien@,wanadoo.fr
}

La géographie sociale s'est préoccupée de longue date des outils d'intervention visant à organiser et à façonner le territoire. Elle s'y est intéressée avec l'objectif de participer à une géographie appliquée, sachant penser la spatialité des phénomènes sociaux mais aussi analyser et organiser leur régulation. Elle a ainsi contribué à développer une réflexion sur l'intervention aménagiste, en tant que dispositif d'action politique, technique et idéologique, régissant l'organisation publique des territoires.

Nous nous proposons dans ce cadre d'interroger l'émergence d'une prospective territoriale locale. Celle-ci constitue en effet un nouvel outil, à la fois pour l'analyse de l'espace et pour la mise en œuvre de nouvelles démarches. Elle appelle ainsi à de nouvelles compétences et à de nouveaux savoirs pour une géographie appliquée : sur les dynamiques temporelles, sur les dynamiques collectives, sur les rapports entre savoirs et pouvoirs ou action. En ce domaine, elle demande en quelque sorte une ingénierie de la construction de territoires, et nous en verrons les formes et les raisons.

Cependant, par delà ses apports méthodologiques et techniques, elle est intéressante dans ses enjeux, dans ses significations et sa portée pour l'intervention publique sur les territoires. La prospective suppose en effet l'articulation entre un savoir technique et localisé, des modalités de participation collective, la gestion et la production d'une symbolique du territoire à travers la définition de l'avenir. Elle produit doublement du collectif, dans la fabrication d'une figure du territoire, de sa cohérence et de son identité, et dans la production collective de cette image. Elle met en place également des formes de maîtrise de cette construction collective, par une expertise adaptée ainsi que par la recherche d'une continuité, et donc d'une nouvelle temporalité. Plus généralement, et c'est l'interprétation que nous voulons défendre ici, la prospective consacre une nouvelle territorialité de l'action publique, où c'est la production plus que la gestion d'un système de relations sociales localisées qui est visée. 


\section{Les originalités d'une prospective territoriale locale}

Les enjeux et les intentions sous-tendant le développement de la prospective peuvent être retracées à travers une mise en perspective diachronique. C'est en effet par le passage d'une prospective nationale $d u$ territoire à une prospective locale des territoires que l'objet prend des formes nouvelles. Certes, une pensée du futur est fréquemment mise en œuvre dans les territoires locaux, mais elle prend cette fois une forme institutionnelle, s'organise et s'outille.

La prospective globale ayant pour objet l'ensemble du territoire national se met en place à partir des années soixante. Les exercices de la Délégation à l'aménagement du territoire (DATAR) et en particulier le fameux «Scénario de l'inacceptable » (DATAR, 1970) constituent l'acte de naissance d'une démarche qui prend ses distances avec la planification et le plan. Il ne s'agit plus alors d'organiser mais bien de fixer des images du futur à partir desquelles définir une politique volontariste. La démarche se spécifie dans ce crochet par le futur en fonction duquel sont déterminées des orientations présentes. Pour autant, elle reste marquée par une pensée progressiste et positiviste, qui hérite à la fois de la prospective des entreprises, de l'approche techniciste et fonctionnaliste de la planification et de l'idéologie volontariste de l'Etat. Elle parvient ainsi à conjuguer une croyance dans la capacité à déterminer les évolutions et une croyance dans la capacité de l'action publique à les infléchir. On a la "conviction qu'il est possible d'éclairer l'avenir grâce à une analyse fine et rigoureuses des données statistiques qui permettent de repérer les tendances lourdes et les possibilités de changement»(F. Plassard, 2002, p. 197), dont les scénarios seront une traduction privilégiée.

Devant les échecs de cette approche prévisionniste et préventionniste et ses insuffisances ou apories conceptuelles dans un contexte complexe et turbulent, la prospective nationale disparaît quasiment du paysage à la fin des années soixante-dix, en même temps que l'aménagement du territoire est remis en cause.

La relance apparaît vigoureusement en tout début des années quatre-vingt dix, avec de nouveaux grands exercices nationaux : l'opération "Prospectives et territoires » s'étend de 1990 à 1995, le programme «Territoires 2020 » lui fait suite en 2000 et «Territoires 2030 » s'amorce dès 2004. La prospective régionale s'est développée à sa suite, à la faveur du premier acte de la décentralisation. Une très forte majorité de régions, on l'évalue à dix-sept ou dix-huit (G. Loinger, 1999 et 2004), a aujourd'hui mené une opération qui par son intitulé à défaut de son contenu se prévaut de manière explicite de la prospective.

D'autres niveaux territoriaux s'en emparent dans le même temps. La démarche « Lyon 2010 » (1985) peut être considéré comme un exercice précurseur en France de planification stratégique appliquée à une agglomération urbaine. Plus tardivement, ce sont les communautés d'agglomération, les pays et d'autres structures intercommunales qui mettent en place de telles démarches, incité en cela par la loi Voynet (Loi d'orientation d'aménagement et de développement durable du territoire, 1999). On voit aujourd'hui des tendances en faveur d'une extension à ces échelles locales intercommunales, si bien qu'Aliette Delamarre (2002, p. 24) évoque une « miniaturisation des terrains ».

Celle-ci n'est pas sans une certaine confusion conceptuelle : certaines collectivités mettent en place des services dédiés, d'autres en adoptent la posture d'anticipation en réfutant l'intitulé, d'autres en utilisent les images positives pour renommer des démarches traditionnelles ou sans consistance. 


\section{Les formes et enjeux de la relance}

L'extension de la démarche est liée à deux phénomènes concomitants : une transformation dans la sphère du pouvoir et une transformation dans la sphère des savoirs. La prospective d'une certaine manière en est une des articulations, un lieu où, dans la médiation du territoire, se jouent leurs rapports respectifs.

Les transformations institutionnelles mettent en cause l'organisation de la décision et plus généralement des rapports entre territoires. En même temps que les collectivités régionales ou locales s'autonomisent politiquement, elles développent dans un contexte devenu concurrentiel et prenant pour modèle d'action l'entreprise, des conduites stratégiques individuées. Une connaissance spécifique, des outils adaptés deviennent en ce cas des enjeux majeurs. La prospective participe à l'autonomisation de l'action, en premier lieu par un apport d'information et de connaissance finalisées ainsi que par les perspectives qu'elle offre d'une stratégie politique à cette échelle. A des collectivités locales nouvellement constituées elle offre cette possibilité d'appréhension de la globalité du territoire, de ce qui la fédère et en définit la cohérence. Les dispositifs d'observation et de veille, de production de connaissance qui l'accompagnent conditionnent l'exercice d'une maîtrise sur cet espace. Ces nouvelles échelles d'autre part posent avec plus d'acuité la question de la citoyenneté. Si la nature et les formes de mobilisation de la citoyenneté sont remises en cause de manière générale par une crise de la représentation, la légitimité du territoire est moins ébranlée que à fonder dans des collectivités en émergence politique. Celles-ci se trouvent face à la nécessité d'offrir une représentation d'elles-mêmes, de manifester de leur existence en tant que cadre collectif, référent d'un attachement et d'une participation.

C'est dans cette dimension politique que l'on peut lire le développement d'une prospective régionale, mais plus généralement de la prospective dans les territoires en recomposition ou même dans les récents exercices nationaux. Lorsque la vision d'une unité s'effrite ou est à faire, lorsque les représentations de, ou les tendances à, la dislocation deviennent trop fortes, comme l'on montré les travaux préparatoires à la première Loi d'orientation sur l'aménagement et le développement du territoire (1992) ou les scénarios de Jean-Louis Guigou (1995), la prospective constitue un moyen de produire une représentation de l'unité. Projetant le territoire dans l'avenir, elle en dessine nécessairement une figure, une personnalité qui la font apparaître comme une totalité et une individualité. A l'indétermination de l'identité présente du territoire, elle oppose la figure du futur, figure virtuelle mais attestant néanmoins d'une existence.

La prospective répond ainsi à la nécessité de trouver et plus précisément de fonder une assise sociale et politique, de structurer des rapports tout en recourant à d'autres moyens pour ce faire que ceux de l'institution ou de l'organisation fonctionnelle.

Par ailleurs, la transformation du contexte institutionnel et politique a pour corollaire la transformation des modes d'aménagement, tant du point de vue des conceptions que des procédures et des démarches opérationnelles. Les lois d'aménagement et de développement $\mathrm{du}$ territoire, les documents de planification et de développement, qu'ils portent sur l'organisation spatiale tels les Schémas de cohérence territoriale (SCOT) ou sur des thématiques plus récentes comme les Plan de déplacement urbain (PDU), imposent des opérations de diagnostic et des démarches de projet. Si ni les unes ni les autres ne peuvent 
faire office de prospective, elles conduisent à mettre en lien information, stratégie et définition d'orientations cohérentes. Le diagnostic conduit à une interrogation sur les méthodes et démarches, en particulier de collecte, d'analyse et de visualisation de l'information. La relance des schémas territoriaux mène à l'élaboration de méthodologie pour une connaissance véritablement stratégique.

On se trouve donc face à un système de contrainte, de nécessité et de volonté : volonté d'autonomie et d'accroissement des capacités d'action, injonction à repenser l'aménagement et l'organisation, nécessité de redéfinition des outils et nécessité de redéfinir, par et pour l'action, le territoire pris comme objet de l'intervention.

En parallèle à cette dimension institutionnelle et politique, la relance de la prospective est liée à ce que l'on pourrait appeler un nouveau rapport sociétal au progrès. Le concept de durabilité impose un mode de pensée projectif et quelle que puisse être la consistance des applications, suppose pour le moins une définition de valeurs territoriales fondamentales, dont on veut assurer la reproduction. L'évaluation et l'impact qui lui sont corrélés, tentent également de prendre en considération les effets, intentionnels ou non, des actions. Incertitude et complexité de l'environnement quant à elles alimentent la recherche de nouveaux outils de pilotage pour un tel contexte, qui trouvent des matériaux méthodologiques dans l'analyse de la dynamique de l'innovation. Celle-ci intéresse dans la mesure où elle apparaît comme une dimension majeure de la croissance économique. Mais elle fonde aussi de nouvelles approches des évolutions, des conditions de l'invention ou de ruptures dans les modes de pensée. L'anticipation dans ce contexte consiste non plus en une projection linéaire de tendance, mais en la recherche de signaux de l'inflexion ou de l'émergence, en la mise en place également d'éléments propres à provoquer ces inflexions et ces ruptures et à fonder l'innovation.

Au niveau territorial, la recherche des conditions de l'innovation devient recherche d'une action sur la culture, au sens large du terme, action sur les croyances et les significations, action également sur les dynamiques collectives : "Un territoire c'est aussi un vécu, une identité, une subjectivité, un système de représentation, de conscience. [...] Et un territoire c'est un cadre de gestion, et de gouvernement de la scène publique, c'est une entité qui se manage, qui se pilote » (G. Loinger et C. Spohr, 2004, p. 26).

Ces quelques éléments permettent de brosser le paysage de la prospective territoriale actuelle. Plus qu'une histoire des procédures, facile à reconstituer par ailleurs, ils permettent de comprendre la redéfinition des contenus de la méthode. Ils permettent également de mettre en exergue des filiations : de l'entreprise, de la planification, du positivisme, de la procédure d'un côté, de la participation et de la culture de l'autre côté.

\section{La prospective territoriale locale, une tentative de définition}

Avant d'en analyser les formes et les conséquences, tentons de préciser le contenu et plus largement la nature de la prospective territoriale locale. Nous avons volontairement repoussé cette définition après la description de son développement pour en faire comprendre le caractère très labile. Si elle peut être entendue au sens très large d'un " regard » (Delamarre, 2002), d'une « attitude anticipatrice » (G. Loinger et C. Spohr, 2004), d'une " philosophie » (F. Goux-Baudiment, 2001) ou d'une pensée du futur imprégnant les actions publiques d'aménagement, nous préférons ici la circonscrire de manière plus précise. Se différenciant d'une activité de l'imaginaire, elle doit être positionnée en tant qu'instrument de 
l'intervention publique, pouvant être isolé d'autres formes d'intervention par des opérations propres, des objectifs explicites et une production en relevant. Occupant rarement un service dédié, sauf dans les régions, la prospective apparaît comme un "exercice » (F. GouxBaudiment, 2000) ou une " démarche » (G. Loinger, 1999) selon la terminologie en vigueur, plus généralement dirons-nous un dispositif, qui contient :

- une dimension cognitive. Il a pour projet non pas une connaissance d'un avenir mais la production de connaissances donnant capacité à anticiper et à piloter les changements.

- une dimension technique et pratique. Un ensemble d'outils et de méthodes qui empruntent aux techniques de projection quantitatives, aux techniques d'animation et de créativité, etc. La méthode des scénarios est certainement la plus usitée.

- une dimension discursive. Le futur se dit, se met en image, se communique et constitue d'une certaine manière un champ autonome des discours sur le territoire. La prospective nous semble indissociable de la projection, c'est-à-dire d'une visualisation explicite, qu'elle qu'en soit la forme, d'une image du futur.

- une dimension opérationnelle et organisationnelle. La prospective en particulier et la conduite de projet plus généralement conduisent à des dispositifs inédits d'organisation de l'intersectorialité.

- une dimension programmatique. La prospective se structure en force de proposition et d'accompagnement en direction de l'arbitrage stratégique et de la mise en œuvre d'actions mais elle n'a pas vocation à s'y substituer.

La plupart des auteurs parlent de quatre familles d'outils et de méthodes prospectifs : la prospective prédictive (les projections de tendances), la prospective descriptive (les visions d'avenir, les scénarios), la prospective débat (la prospective participative et/ou en club de réflexion sur l'avenir) et la prospective préventive (la veille). Les professionnels du développement territorial qui initient un exercice de prospective ont tendance à se saisir de ces familles successivement. La veille permet de cerner les enjeux à relever; les moins complexes de ces enjeux (la démographie dans une certaine mesure, etc.) - dont on peut imaginer qu'ils évolueront selon une dynamique tendancielle - sont projetés à l'aide de méthodes économétriques ; les plus complexes des enjeux sont embrassés par la construction de visions d'avenir, qui s'organisent généralement autour de l'élaboration de scénarios. Les scénarios exploratoires sont les premiers à être élaborés en ateliers d'experts. Les acteurs du territoire sont associés à l'élaboration des scénarios normatifs qui permettent de passer à la dernière famille d'outils. Sur la base de ces extrapolations et explorations, le débat est initié et orienté vers l'action. Lorsqu'on le confronte aux logiques institutionnelles et organisationnelles, ce cheminement vertueux se révèle bien peu évident à suivre. Il s'agit d'autant plus d'une impasse que l'on s'aperçoit qu'il existe plusieurs scènes prospectives sur un territoire - portées par les chambres consulaires, les collectivités territoriales, les associations, etc. - qui se spécialisent dans l'une ou l'autre des formes de prospective. Ce qui ne signifie pas pour autant qu'il n'existe aucun emprunt méthodologique aux autres familles d'outils. Nous assistons, par analogie avec la division des tâches en économie à une division des tâches prospectives sur les territoires locaux avec une phase d'innovation, une phase de maturité et une phase de déclin. C'est ainsi qu'une institution/acteur du développement d'un territoire, loin d'être l'organisme prospectif de référence, va se spécialiser dans une tâche prospective laissée vacante par les autres acteurs, jusqu'à épuiser cette tâche (dans le rapport à la stratégie essentiellement). Il lui faudra alors renouveler sa posture et probablement se saisir d'une autre famille d'outils prospectifs. Cet enchaînement n'est pas automatique et peu prendre plusieurs années bien que les collectivités territoriales, c'est un nouvel enjeu, essaient de systématiser la pensée du futur du territoire. 
Il n'est pas toutefois dans notre propos ici de décrire l'émergence d'une nouvelle branche de l'aménagement et d'en montrer la diversité de son instrumentation. On a vu en quoi elle résultait de changements de contexte et de modes de pensée. De manière plus fine et dans une perspective d'analyse cette fois de ses conséquences, essayons d'en montrer les implications dans l'action publique. Par ses contenus, l'exercice de prospective apparaît comme un révélateur de la transformation d'une territorialité, nous en avons vu quelques éléments cadre. Mais il en constitue aussi un moteur. C'est dans ce triple plan, de la décision, de la conceptualisation et de l'instrumentation et à travers une analyse des pratiques, que nous voulons examiner l'hypothèse d'une territorialité nouvelle, d'un mode de construction nouveau du territoire.

\section{Les formes de la territorialisation issue de la prospective}

\section{Le rapport au temps}

L'exercice de prospective, fondamentalement, introduit une dimension temporelle. Celle-ci relève bien évidemment de l'objet et de la démarche de l'action. Mais elle nous semble plus importante encore en ce qu'elle construit une temporalité territoriale. Celle-ci peut être posée en regard de territorialités contemporaines que l'on dit éphémères, mobiles et éclatées ( $\mathrm{J}$. Viard, 1994, G. Di Méo, 1996, MC. Fourny et A. Micoud, 2002). Elles montrent que l'histoire des individus ne s'inscrit plus dans un territoire unique et stable et, inversement, que la mémoire longue du territoire ne fait plus l'objet d'un processus spontané de transmission inter-générationnelle.

Par rapport à cette perte d'historicité, la prospective réintroduit une durée. Elle inscrit la collectivité dans une permanence ; la projection dans l'avenir témoignant que, par devers la mobilité et l'instabilité des sociétés qui le constituent, le territoire conserve une existence. La substantialisation de l'espace considéré se fait grâce à ce double mouvement, de figuration et de durée. La figure qu'implique la définition d'une vision de l'avenir nécessite en effet de dresser les contours d'une identité. Elle a en cela valeur performative : en énonçant et formalisant son objet, en le dénommant et en le qualifiant dans une représentation globalisante, elle lui donne corps, et en atteste l'existence. La projection démontre par ailleurs une permanence, elle dessine une temporalité supérieure à celle des individus qui y résident. De ce point de vue, la prospective constitue un pendant de la patrimonialisation. Projection dans le futur et mise en scène d'une histoire fondent tous deux une dimension temporelle intrinsèque, quasi a-sociale. C'est dans la continuité, dans ce lien entre passé, présent et avenir que peut se construire le sentiment d'une personnalité, d'une spécificité inscrite dans un espace plutôt que dans sa société (G. Vinsonneau, 2002). Grâce à ce processus, le territoire acquiert un statut d'objet collectif, singulier et autonome.

A la différence du patrimoine, la prospective toutefois contient une dimension utopique. En dressant l'image d'un futur aussi bien attractif que possible, elle peut constituer un levier pour l'action, et s'avérer non seulement une connaissance sur les processus, mais aussi un agent de structuration sociale. Ce rôle se manifeste de plusieurs manières :

- la prospective tout d'abord conditionne la formation d'une mobilisation. Tout comme les images du passé, la vision du futur permet en effet d'opposer aux situations présentes, à une crise implicite ou explicite, un meilleur possible. Mais là où le patrimoine recourt à la nostalgie, la prospective évoque la capacité d'agir. A la 
différence également de la prévision qui postule un avenir irréductible, la prospective en réfère à la maîtrise et à la volonté. La construction du territoire comme un objet doté d'une permanence s'intègre à cette figure de l'action : ainsi mis à distance, le territoire peut devenir non plus seulement l'espace de la collectivité, mais l'objet commun de l'action collective. Il peut dès lors s'inscrire dans un discours sur la responsabilité où, selon la rhétorique en usage, «l'avenir est l'affaire de tous ».

- il participe d'une dynamique collective de changement. Renvoyée à un devenir forcément meilleur, la collectivité se trouve dotée d'une image positive d'elle-même. La prospective devient en ce cas un outil d'intervention sur la psychologie collective. Il s'agit parfois d'une opération se limitant à la communication interne ou au marketing, visant à produire des images attractives. Il s'agit aussi de s'en servir pour introduire de nouveaux modes de pensée, faciliter l'appropriation du changement et de l'innovation, dans la mesure où, à la différence là encore de la patrimonialisation, la prospective intègre les mutations dans la représentation du territoire. Ce travail de conceptualisation de l'avenir relève alors quasiment d'un travail thérapeutique : il permet aux « territoires en dépression de s'en sortir» (A. Delamarre, 2001, p. 21). Cet enjeu n'exempt pas la prospective des risques de manipulation d'une personnalité collective. On retrouve ainsi dans la terminologie employée, dans les références à un esprit positif et à la recherche de performance, bien des similitudes avec les formes actuelles du management de la ressource humaine dans les entreprises. A minima, la prospective peut se voir restreinte à cet objectif de communication, et utilisée comme une opportunité pour une production de discours, sans plus de prolongation dans une action.

La construction temporelle du territoire pour autant ne fait pas apparaître une réelle définition d'une trajectoire territoriale, qui viendrait mettre en perspective le passé du territoire avec son avenir. Avec le patrimoine et la prospective, l'ancrage temporel se fait dans deux directions à partir du présent, mais dans une pensée et une opérationnalité qui les disjoignent. Jamais, dans les expériences observées ou relatées, on ne trouve une mise en perspective historique. La prospective relève moins de ce fait de la construction d'un récit, au sens que peut lui donner Ricœur, que d'un discours sur le présent. Par rétroprojection en quelque sorte, la vision du futur est reliée au présent. Elle conduit à définir et à rendre explicite le commun, à formaliser un nous territorial, mais assez paradoxalement cette identité apparait intemporelle, sans historicité. La raison en est organisationnelle et culturelle : la prospective ressortit au champ de la planification et de l'action, aussi bien dans sa pensée que dans sa mise en œuvre. Le discours sur le territoire qu'elle produit est un discours d'insertion dans le changement : l'identité mise en exergue est une identité pragmatique, opérationnelle, elle vise à montrer la place du territoire dans un monde en mouvement. Le commun est décliné ainsi dans une culture collective, savoir-faire, pratiques, comportements, attitudes. Il fait ressortir des qualités collectives plutôt que des biens, dans un processus de personnalisation classique : «Grenoble est innovante, cosmopolite et montagnarde ». Plus encore, il sélectionne ces traits en fonction de leur valeur, les décrit et les qualifie sur des critères de valeur, voire parfois d'utilité économique. Le procédé a pour avantage de montrer la pertinence et la potentialité de spécificités culturelles. Mais, s'inscrivant dans un autre registre de construction de l'identité que celui de la patrimonialisation, il conduit à l'en dissocier.

Construction du territoire dans le temps et par le temps, la prospective s'insère ainsi dans le champ des politiques publiques temporelles. Celles-ci sont le plus souvent aujourd'hui réduites aux politiques de synchronisation des temporalités publiques et individuelles et se focalisent sur les bureaux des temps ou la régulation des mobilités (JY. Boulin, F. Godard et al., 2003). Or l'une et l'autre sont complémentaires. Avec le patrimoine, elles manifestent 
d'une intervention sur le temps destinée à produire la cohérence et l'unité qui ne résultent plus des inerties spatiales, des effets de la proximité ou de la stabilité dans le temps d'éléments matériels. A cette incapacité de l'espace à instaurer des liens sociaux, on oppose la conjonction présente des temporalités, et des figures référentielles d'une stabilité dans le temps.

Objet de politiques publiques, le temps devient également objet de connaissance, dimension possible d'une maîtrise. Et si la prospective ne relève plus d'une croyance dans le progrès, elle procède encore d'une croyance dans une capacité, collective ou experte, à maitriser des évolutions dans le temps : "l'avenir est l'objet d'une création collective » (G. Loinger, 2004, p. 18). Mais l'objet de la maitrise devient celui de la conduite dans le temps. La posture n'est finalement pas moins volontariste que celle prévalant dans l'aménagement fonctionnaliste. Elle ne passe toutefois par la même instrumentation ou les mêmes ressources cognitives.

\section{Le rapport au collectif}

De premiers éléments de modalités d'une construction collective du territoire sont directement corrélés à la dimension temporelle. Le territoire projeté devient l'objet commun et intentionnel fédérant le collectif. Il représente tout autant un vecteur de mobilisation que le produit de la démarche : les déclarations telles que "nous allons devenir un territoire » en sont représentatives (A. Delamarre, 2001, p. 22). La démarche prospective par ailleurs substantialise le collectif, nous l'avons évoqué, que ce soit par une projection unifiante et configurante ou par la définition d'une personnalité.

Ces enjeux et ces fonctions de la prospective ne sont guère différents nous semble-t-il, du projet. Outil d'une gouvernance des acteurs, supports d'une figuration symbolique et existentielle, les deux démarches produisent le collectif qu'elles se sont données pour objet. La prospective toutefois se distingue par un type de structuration original et poussant encore plus loin l'intégration que réalise l'aménagement de projet.

Cette structuration intervient au travers du processus mis en œuvre. Reprenons les deux grands types de prospective habituellement distinguées : une prospective experte et une prospective participative. La première privilégie la production d'une connaissance sur le territoire, sur les processus de transformation, et mobilise essentiellement spécialistes des différents domaines et spécialistes des techniques d'expertise en prospective. La seconde vise à la définition collective d'une figure de l'avenir commune, dans un processus chemin-faisant. Elle installe des lieux de débat, des scènes ou forums participatifs dans lesquels se travaillent collectivement les représentations du territoire et où l'on met en question explicitement l'identité du territoire. Ces deux types, d'évidence, relèvent de deux conceptions de la démarche et engagent différemment le rapport entre élus et population comme le rapport au temps ou à l'action. La démarche participative procède d'une conduite fédératrice d'acteurs diversifiés, mobilisés par une perspective commune qui se veut elle-même l'émanation en continu du travail collectif (M. Callon, P. Lascoumes, Y. Barthe, 2001). La démarche cognitive relève quant à elle d'une évaluation de tendances dont les scénarios sont la traduction la plus connue. Elle fait appel pour une part à un important outillage technique de production et d'analyses d'informations objectives : observatoires, SIG, modélisation. Elle utilise d'autre part un ensemble de méthodes d'exploration procédant d'une synthèse d'évolutions sectorielles à différentes échelles. La première, pour la plupart des observateurs de la prospective, correspond à une démarche de gouvernance (P. Destatte, 2003, F. Ascher, 
1998). Elle en constitue un outil, un outil appliqué à une gestion du changement et à une mise en œuvre collective d'une conception du territoire.

Pour autant les méthodes employées par la prospective exploratoire ne permettent pas d'opposer les deux figures et de voir dans l'une un outil d'ordre politique, dans l'autre un outil technique d'une nouvelle expertise. La prospective exploratoire en effet doit horizontaliser des expertises sectorielles. Elle fait appel de ce point de vue à une perspective interdisciplinaire, avec toutes les difficultés que celle-ci peut rencontrer. Mais en même temps, elle doit se dégager du diagnostic, intégrer les dynamiques collectives et culturelles. Par ailleurs les méthodes d'exploration les plus couramment utilisées restent très largement empiriques, la connaissance objective s'y appuie sur une connaissance fine et qualitative d'un milieu économique, de ses comportements et de sa culture. Elles demandent des qualités d'intuition, d'interprétation et d'agencement de ces données d'une autre nature que les qualités expertes classiques. Le produit des analyses par ailleurs sollicite de nouvelles formalisations. Dans des exercices de prospective qui accordent une importance majeure à la communication, la représentation de la figure du territoire, sous une forme cartographique, iconographique ou discursive devient essentielle (B. Debarbieux et S. Lardon, 2003). Le savoir-faire ne consiste pas là tant dans l'agencement de connaissances spécialisées que dans l'expression de leur projection sur le territoire. Les représentations graphiques affectionnées par les scénarios de l'aménagement du territoire en sont des exemples significatifs.

De nouveaux objets de connaissance et d'intervention apparaissent également dans ces opérations. La question de l'identité devient ainsi une thématique en soi, non pas seulement dans un objectif de valorisation, mais aussi d'évaluation de ses effets sur les processus de changement. Objectivée, elle est portée en débat, déconstruite et évaluée.

Les procédures participatives développent quant à elles largement des forums où interviennent conjointement habitants, spécialistes, techniciens de l'aménagement, élus parfois. Les thèmes mis en débat collectif sont d'ordre existentiel : ils renvoient à des qualités, des référents identitaires, des objets transversaux et à forte dimension subjective. Ce type d'intervention n'oppose pas comme dans la plupart des procédures l'expertise à la subjectivité des habitants, ou la connaissance intuitive d'acteurs locaux à l'objectivité de consultants externes : il confronte des savoirs, des formes d'expérience et de sensibilité différentes. Il serait abusif de parler d'équivalence, les hiérarchies et les rapports de pouvoir sont bien trop ancrés pour cela, néanmoins le dispositif instaure un cadre d'une mise en relation: experts et habitants exposent des points de vue dont chacun a le même intérêt en regard de l'objectif de définition du territoire commun. La production d'une image du devenir relève en ce cas d'une vision dite holoptique, par opposition à la vision panoptique de l'aménagement fonctionnaliste ; vision holoptique qui, dit Pierre Lévy, « doit offrir une perception complète d'un espace, où chacun, par son expérience et ses connaissances peut les ajuster et les coordonner aux autres » (P. Lévy, 1995, p. 25).

Si autant les démarches participatives que les méthodes exploratoires demandent des transferts et des agencements de savoirs, l'enjeu essentiel de la prospective réside cependant dans sa capacité à articuler la production collective issue du débat, avec la production objective des experts. C'est dans cette articulation que la prospective peut dépasser l'utopie ou la stratégie pour devenir un dispositif opératoire intégrant le souhaitable et le voulu avec le possible ou, autrement dit, intégrant la rationalité et la dynamique collective. C'est dans cette articulation aussi que la prospective locale peut être proprement territoriale. On entend dans ce caractère non l'étendue ou l'objet du projet, mais une dynamique sociale et 
culturelle, intégrant dans les rapports entre acteurs et produisant à partir des rapports entre acteurs, un rapport au territoire, c'est-à-dire un sentiment d'une identité, d'une appartenance et d'un objet communs, d'une responsabilité, référés à un espace.

Ce mode de structuration fait de la prospective plus que cet outil d'une gouvernance habituellement décrit. Les processus mis en oeuvre sont de nature à faire émerger une construction relevant d'une intelligence collective territoriale. Si l'intelligence collective constitue « une intelligence distribuée, coordonnée, qui aboutit à une mobilisation effective des compétences » (P. Lévy, 1994, p. 29), l'intelligence territoriale demande à notre avis une production de connaissances organisée sur l'espace local, mais aussi une collaboration des acteurs mobilisant leur territorialité, s'appuyant sur un référent territorial, sur des significations partagées, qui les mobilisent individuellement et les fédèrent collectivement. La prospective en offre un support possible, par le double registre participatif et cognitif qu'elle sollicite ainsi que par la pluralité des statuts du territoire qu'elle met en jeu. Celui-ci y est construit autant en étendue qu'en médiateur, objet et en final, produit.

Il en résulte des besoins de compétences nouvelles, qui ne sont pas sans pouvoir éveiller l'attention d'un géographe. La mise en œuvre de la prospective sollicite une capacité à gérer de la relation, à créer du débat, et à faire émerger un bien commun. Elle appelle à un savoirfaire dans l'intégration des significations dans l'action, dans une gestion des aspects symboliques et culturels qui ne relève pas de la manipulation ou de la communication. Elle demande finalement une intervention non pas de l'ordre de l'organisation spatiale ou de la médiation sociale, mais portant plus précisément sur des territorialités, c'est-à-dire, sur un processus collectif, spatial, organisationnel et identitaire.

\section{Le rapport entre territoires}

Le rapport entre territoires engage la dimension politique de la circonscription considérée : en quoi la prospective engage-t-elle la construction institutionnelle et peut-elle transformer les relations entre mailles et niveaux d'intervention?

Examinée d'un point de vue interne, elle représente pour une (grande) part un dispositif de légitimation de l'action publique. La substantialisation que nous avons évoquée naturalise en effet l'espace de l'intervention et de ce fait légitime l'espace que représente l'élu. Cet aspect n'est pas sans expliquer son attrait et sa réduction parfois à des opérations de communication. Il en fait un outil pertinent pour toute situation de crise institutionnelle, qu'il s'agisse de structures territoriales émergentes, des régions dont l'identité est à fonder, de départements ou de communes remises en question, ou même d'un territoire national à l'unité menacée.

Dans le même temps, elle constitue un agent de transformation de l'action publique. Sauf à la réduire strictement à un diagnostic exploratoire, elle mobilise, fédère, participe d'une structuration territoriale. D'autre part, le mode de construction collective, dans la conjonction des savoirs que nous avons évoquée, n'est pas sans rendre possible une redéfinition des rôles entre acteurs techniques et acteurs élus, pour l'heure porteurs des opérations. La compétence dans la conduite du changement, la capacité à la mise en débat, la mise en figure, la définition d'un futur, relèvent bien d'un art du gouverner. Or la prospective les transfère dans la sphère technique et en fait une dimension opérationnelle. Les techniciens acquièrent de ce fait de nouvelles capacités d'orientation politique, entrent dans le domaine constitutif de l'élu en développant et rationalisant la compétence fondatrice mais intuitive de ces derniers à 
exprimer le collectif qu'ils représentent. En l'absence de portage des élus, on les voit alors parfois fort mal à l'aise, pourvus d'une arme dont ils ne peuvent ou ne veulent pas se saisir. Ou alors à l'inverse, ils l'utilisent dans les jeux de ruse régissant les relations entre techniciens et élus : l'objectivation du futur peut devenir force de pression ou instrument d'une nouvelle pédagogie. La prospective infléchit ainsi les modes d'action de l'élu, joue sur les systèmes de représentation, tout en confortant le territoire comme fondement de la représentation.

Considérée d'un point de vue externe, l'existence de prospectives territoriales locales pose plus encore que la planification le problème de l'articulation des territoires et de leur capacité à concevoir simultanément leur singularité et leur appartenance à d'autres territoires. La prospective par le haut pouvait à la rigueur entretenir la conviction d'un avenir commun. Avec la prospective par le bas, certaines vertus démocratiques sont susceptibles de progresser, mais aussi avec elles des risques d'éclatement des visions d'un intérêt collectif. La multiplicité des prospectives locales interroge par ailleurs l'intérêt et la place d'une prospective nationale. Le problème est à la fois vertical, dans l'emboîtement des échelles et niveaux de prospective, et horizontal dans l'intégration ou la prise en compte des voisinages. Or cet axe est sans doute celui qui connaît le moins d'innovation. Les descriptions des grandes figures emblématiques de la prospective territoriale n'évoquent pas le sujet. Dans nos observations locales, les prospectives locales se superposent ou se multiplient, le plus généralement en s’ignorant.

Les raisons en sont nombreuses. On peut incriminer la nouveauté des démarches, dont la mise en œuvre et les conséquences n'ont pas eu la durée nécessaire pour donner lieu à des confusions éventuelles. Plus sérieusement, l'opération reste aux mains de collectivités locales, pour qui elle représente un moyen nous l'avons vu, d'affirmer l'existence de leur territoire. De ce fait la connaissance mobilisée ne se restreint pas à la maille concernée : l'échelle considérée est celle des phénomènes non de la maille administrative. Mais l'espace de référence de la stratégie reste bien celui de la collectivité locale. Les jeux géostratégiques restent ainsi classiques : de concurrence, d'alliance stratégique éventuelle, de recherche d'une spécificité. La prospective de ce point de vue offre la possibilité d'un meilleur positionnement, d'une évaluation plus fine des rapports avec d'autres territoires. Mais elle demeure un outil au service du territoire qui l'utilise, la prospective partagée apparaissant comme un horizon fort lointain!

\section{Conclusion}

On peut interpréter la prospective territoriale comme un dispositif gouvernantiel de conduite et de production de territoire. Elle prolonge, dans le champ de la gestion et de la production d'une connaissance opératoire les principes mis en œuvre dans le champ politique, à travers la participation et la production, s'insère dans les nouveaux référents managériaux de l'aménagement. Pour la géographie sociale, elle nous semble intéressante en tant qu'outil et mode de la construction contemporaine des territoires. Cette construction s'effectue à travers les significations, dans la structuration et la mobilisation des acteurs. Mais elle trouve là à s'étendre dans la dimension stratégique, gestionnaire et cognitive, par la mobilisation de savoirs et l'articulation avec des méthodes d'objectivation et de rationalisation : territoire signifiant, territoire stratégique et territoire intelligent sont produits conjointement. Elle étend également la problématique de la maîtrise de l'espace à cet objet nouveau de sa dimension temporelle. Ces enjeux sont bien compris, et expliquent l'attrait pour la démarche. Mais les 
freins, organisationnels, politiques et culturels, sont nombreux, liés notamment au fait qu'il s'agit d'un outil de reproduction de la légitimité et de l'existence du territoire, peu apte à d'autres rapports politiques.

Les enjeux problématiques et professionnels d'un tel champ en géographie sont dès lors aisés à cerner. La géographie appliquée a une place à acquérir dans la connaissance et la formation d'une compétence à cette maîtrise de la dimension temporelle et territoriale d'un collectif. Elle trouve là également un matériau pour requestionner l'action publique dans ses rapports au territoire, pour explorer les nouvelles dimensions stratégiques et opérationnelles des identités, des savoirs et des imaginaires.

\section{Bibliographie}

Ascher F., La République contre la ville : essai sur l'avenir de la France urbaine, Paris, L'Aube, 1998.

Boulin JY., Dommergues P. et Godard F. (dir.), La nouvelle aire du temps, Paris, L'Aube, 2003.

Boutinet JP., Anthropologie du projet, Paris, Presses universitaires de France, $2^{\text {ème }}$ édition, 1993.

Callon M., Lascoumes P. et Barthe Y., Agir dans un monde incertain, Essai sur la démocratie technique, Paris, Seuil, 2001.

Candau J., Mémoire et identité, Paris, Presses universitaires de France, 1998.

DAtar, Une image de la France en l'an 2000. Le scénario de l'inacceptable, Paris, La Documentation Française, 1970.

Debarbieux B. et Lardon S. (dir.), Les figures du projet territorial, Paris, L'Aube, 2003.

Delamarre A., La prospective territoriale, Paris, La Documentation française, 2002.

De Jouvenel H., « La prospective appliquée aux territoires », Pouvoirs Locaux, n50, 2001, p. 40-44.

Destatte P. et Van Doren P. (dir.), La prospective territoriale comme outil de gouvernance, Charleroi, Wallonie, Institut Jules Destrée, 2003.

Di Meo G. (dir), Les territoires du quotidien, Paris, L'Harmattan, 1996.

Fourny MC. et Micoud A., "Représentations et nouvelles territorialités : à la recherche du territoire perdu », Debarbieux B. et Vanier M. (dir.), Ces territorialités qui se dessinent, Paris, L'Aube, 2002, p. 31-52.

Goux-Baudiment F., Quand les territoires pensent leurs futurs, Paris, L'Aube, 2001.

Goux-Baudiment F., Donner du futur aux territoires, Lyon, CERTU, 2000.

Guigou JL., Une ambition pour le territoire, Paris, L'Aube, 1995.

LeVy P., L'intelligence collective. Pour une anthropologie du cyberespace, Paris, La Découverte, 1994.

LeVy P., «Cyberespace et démocratie, Pour l'intelligence collective », Le Monde Diplomatique, octobre 1995, p. 25.

LoInger G. (dir.), La prospective régionale, de chemins en desseins, Paris, L'Aube, 2004.

Loinger G. et Spohr C., Prospective et planification territoriale. Etat des lieux et propositions, Paris, DRAST, DGHUC, 2004.

Loinger G., Un état des lieux de la prospective régionale en France. Assises de la prospective, Paris, Université de Paris Dauphine, OIPR, 8-9 décembre 1999.

Plassard F., "Une approche rétrospective de la prospective: "le scénario de l'inacceptable » », Géocarrefour, vol 77, n², 2002, p. 197-214.

VIARD J., La société d'archipel ou les territoires du village global, Paris, L'Aube, 1994. 
Vinsonneau G., L'identité culturelle, Paris, A. Colin, 2002. 